\title{
Neuropsychobiology 1984;12:270
}

\section{Author Index}

Ahluwalia, P. 217 Albani, G.F. 112,260 Amabile, G. 260 Ananth, J. 138 Andersen, P.H. 1 Ansseau, M. 255 Astrup, C. 101

Baron, M. 206 Bechar, M. 106 Belmaker, R.H. 96 Bhat, A. 9 Bradford, D. 249 Brambilla, F. 78 Brauman, H. 19 Brouwer, GJ. 4 Burla, F. 260

Carlton, P.L. 158 Carroll, J.D. 122 Cerfontaine, J.-L. 255 Cicardi, M.C. 112,260

Decker, H. 188 DeCuyper, H. 211 DeWitte, P. 73 Dimpfel, W. 188 Dittrich, A. 27 Doumont, A. 255

Ehlers, T. 48 Eisemann, M. 201 Engelsmann, F. 138 Erdmann, G. 143

Facchinetti, F. 78 Faltus, F. 34 Fattapposta, F. 260 Fink, M. 173 Fyer, A. 224

Geerling, F.C. 34 Geisler, A. 1 
Genazzani, A.R. 78 Gerhard, U. 39 Ghose, K. 122 Gil-Ad, I. 106 Görtelmeyer, R. 196 Gregoire, F. 19 Grof, P. 86 Gruen, R. 206 Gualtieri, C.T. 229

Heinze, H.-J. 60,68 Hering, R. 106 Hicks, R.E. 229 Hindmarch, I. 244 Hjalmarson, A. 55 Ho, B.T. 16 Hobi, V. 39 Holmesland, P. 101 Hubain, P.P. 23 Janke, W. 143 Jerushalmy, Z. 93

Kalix, P. 127 Kerkhofs, M. 19 Kleindienst-Vanderbeke, G.

238 Klieser, E. 152 Knorring, L. von 201 Koch, J. 55 Köchers, S. 143 Koumakis, C. 23 Krijzer, F. 249 Küfferle, B. 183 Künkel, H. 60,68 Kuny, S. 27

Laron, Z. 106 Last, U. 96 Lavie, P. 209 Lazzari, R. 260 Lehmann, E. 115, 152 Liebowitz, M.R. 224 
Linkowski, P. 19 Luckner, N. von 27

Mandel, B. 96 Mantanus, H. 255 Markowitz, J. 224 Marks, V. 9 Mathew, R.J. 16 Matousek, M. 55 Maurer, M. 27 Mayo,J.P., Jr. 229 McGrath, PJJ. 224 Mendlewicz, J. 19,23 Miettinen, P. 134 Modai, I. 93 Monittola, C. 78 Morocutti, C. 112,260 Mould, G. 9 Münte, T.-F. 60

Oreland, L. 201

Paykel, E.S. 9 Perez-Reyes, M. 229 Perris, C. 201 Perns, H. 201 Peterson, I. 55 Petraglia, F. 78 Petsche, H. 183 Pockberger, H. 183 Pozzessere, G. 260 Praag, H.M. van 211

Quitkin, F.M. 224

Rabkin, J.G. 224 Rappelsberger, P. 183 Rathbone, M.P. 86 Ree, J.M. van 4 Ritter, M. 48 Rizzo, P.A. 112,260 
Rosenbaum, M. 96 Rousseau, J.-C. 255 Rowan, P.R. 9

Scarone, S. 78 Scholz, M. 60 Scholz, O.B. 115 Segal, S. 209 Singhal, R.L. 217 Snelder, M. 249 Spadaro, M. 112 Spehr, W. 265 Stemmler, G. 265 Stewart, J.W. 224 Stumpf, C. 179 Subhan, Z 244 Taylor, D.L. 16 Terschlüsen, B. 143 Thau, K. 183 Timsit-Berthier, M. 255 Tyano, S. 106

Van Wettere, J.-P. 19 Vartio, T. 130 Verho, E. 130 Verhoeven, W.M.A. 4 Verstraeten, D. 211 Viukari, M. 130,134

Weinman, M.L. 16 Weizman, A. 106 Weizman, R. 106 Werstiuk, E.S. 86 Wied, D. de 4 Winter, M. 115 Woggon, B. 27 Wolfrum, C. 152

Zemishlany, Z. 93 Zimmermann, P. 196 